\title{
Design of the Collection and Transmission System of GPRS Wireless Indicator Diagram Oil Yield Measurement
}

\begin{abstract}
GPRS wireless indicator diagram oil yield measurement collection and transmission system" is a high-tech product integrated with advanced computer technology, micro-signal sensing technology, EMC technology, data information processing technology, low power consumption technology and internet communication technology. Based on GPRS communication mode, within pre-defined sample collection time, this system collects an oil well's indicator diagram, strokes, times of strokes, current, voltage and other parameters when the oil well is running and stores these parameters in a large-capacity flash memory. While meeting the requirement of data upload, this system transmits collected data to the monitoring center by means of GPRS communication. Upon the system host expert software's intelligent analysis and computation, the system could offer relevant parameters of the oil well such as the liquid yield, working status and operating state and real-time display of the GPRS communication status collected from each oil well's terminal network, giving timely alarm against abnormal data collected from each well.
\end{abstract}

Ying Zhang
Keywords-GPRS; indicator diagram oil yield measurement

\section{SYSTEM FUNCTION}

\section{A. Terminal data collection}

1) Real-time data collection: the collection terminal could offer real-time collection of each well's load, displacement, 3-phase current, 3-phase voltage, active power, power factor and times of stroke through various sensors and transducers, the collection frequency is more than 144 times each time of stroke at the actual precision of higher than $0.5 \%$;

2) Storing the collected data: the collection terminal always stores all the complete data collected from last time of stroke so that the backstage could have improved transmission efficiency when extracting the data;

3) Uploading of collected data: the collection terminal connects to the internet through GPRS wireless data transmission module and uploads data to the front end processor or server after packaging the data in specified form;

4) Packaging the collected data: the collection terminal's data upload is the internet transmission in the form of data packet. Before uploading, the collected data has gone through two times of packaging, firstly under the Modbus RTU protocol and then by the TCP/IP protocol. Of course, the backstage computer would have a corresponding unpacking process when receiving the data.

5) Field data collection by portable computer: the collection terminal is a field data collection by portable computer offering a serial communication interface. Though management procedures, the portable computer's management program could transmit the collected data to the server network database through local area network (LAN).

\section{B. GPRS wireless data transmission}

Through China Mobile's GPRS wireless data transmission, the system could realize the upload of the actual measurement data by the collection terminal; it could transmit the field collection data to the backstage front end processor or server in a more reliable manner.

\section{Backstage remote control}

Through the control and management program of the front end processor or server, remote control over the collection terminal can be realized in terms of collection frequency, clock calibration and fixed-time upload, etc.

\section{Backstage data management}

On a fixed-time basis, the backstage computer could passively receive or actively collect the current actual measurement data packet from each well of the front end. Then the backstage computer would breakdown the data packets and stores them into the server's internet database in a format meeting the requirement of the indicator diagram oil yield measurement on data. Besides, all actually measured data on site collected by the portable computer can also be downloaded into the internet database.

\section{E. Graphic display print}

In The operation terminal of the LAN, the actually measured indicator diagram, current curve, voltage curve, power curve and power factor curve left in the internet server database by display print can be inquired through the management program.

\section{F. Failure alarm display}

The front end processor or server system management program could offer real-time display of the collection terminal network' GPRS communication of each well and show failure alarm reminding and offer timely alarm reminding against abnormal data collected in each well.

\section{SYSTEM COMPOSITION}

The system mainly comprises of the computer, data collector, GPRS wireless data transmission device and various sensors. 


\section{SYSTEM HARDWARE}

\section{A. Installation of sensors}

1) Loading pressure sensor installation: the loading pressure sensor is installed on the beam hanger connecting the oil extractor's polished rod and horse-head and is used to measure the polished rod load of the oil extractor.

2) Angular displacement sensor installation: the angular displacement sensor is installed on the walking beam and used to measure the angular displacement of the oil extractor's walking beam.

3) Current sensor installation: the current sensor is installed in the power distribution box of the oil extractor. It uses cables to connect the detected current signals with the controller RTU of the oil extractor.

4) Detection of voltage: through cables the $380 \mathrm{~V}$ or $660 \mathrm{~V}$ voltage signals are transformed to $24 \mathrm{~V}$ voltage signals which would be connected with the oil extractor's controller RTU.

5) Wellhead pressure sensor installation: the wellhead pressure sensor could detect wellhead casing pressure and wellhead pressure. Through cables the pressure signals could be connected with the oil extractor's controller RTU.

\section{B. Installation of the collection terminal cabinet}

Many locations can house the collection terminal cabinet and the actual installation location could be selected according to the actual status on site.

\section{SYSTEM SOFTWARE}

The system uses the solution with front end collection terminal: the GPRS communication module uses UDP transmission mode, presenting fast internet connection and efficient transmission. As the GPRS network charges according to the flow, this way would save some cost. Backstage reception: a server with fixed public IP would be allocated as the system's fixed gateway. By means of the interface mapping software or the Windows 2000 Server operating system's own interface mapping function, the IP address and interface of a computer in the oil field LAN's are mapped to the gateway server. The interface mapping function could allow a machine in the internal network to offer WWW service to the outside. The interface mapping function is to map a false IP of a host to a true IP. When users visit a certain interface of the host offering mapped interfaces, the server would forward the request to an internal host which offers such specific service; using the interface mapping function, multiple interfaces of a machine with true IP could be mapped into different interfaces of different internal machines.

\section{A. The installation and configuration of SQL Server database software}

The SQL Server database software is installed on a computer with fixed IP in the LAN and this computer would be used at the system's database server so that the data center could store the received data in the SQL Server database and facilitate the access of other software into the database server.

\section{B. Installation and configuration of data remote transmission software}

The data center software is installed on a computer with fixed IP in the LAN and this computer will be used as the system's server. As the system server's IP address and interfaces are mapped in the oil field gateway server, this system server could smoothly receive relevant data of the oil well collected by remote GPRS. Additionally, the collected data would be stored in the SQL Server database server in the form of SQL Server data form specified below. Such functions as data collection, data storage, graphic display, alarm judgment and sending alarm information could be realized. The functions are described below respectively.

(1) The set-up of reported time interval and of the data collection item;

(2) Manual remote collection of real-time indicator diagram data;

(3) Automatic report of remote real-time indicator diagram data;

(4) Automatic data storage to facilitate the analysis of yield measurement expert software;

(5) Inquiry, browsing and printing of the indicator diagram, current diagram and voltage diagram.

\section{Yield measurement expert software}

The yield measurement expert software's data reading and storage shall meet the required form of database by the data remote transmission software, ensuring that all data could be safely read, modified and stored in the SQL Server database.

This software could complete such functions as system configuration, data calculation, data analysis, data inquiry and indicator diagram display, etc.

1) System start and log-on

The expert software's log on to the interface is subject to password control. Only authorized users could enter into the system to ensure safe operation of the system.

2) The functions and characteristics of the system

(1) System set-up: this menu item function is used to configure the database server's IP address, user management, division management and other initial configurations.

(2) Basic data: this menu item function is used to import the data of relevant oil well and oil extractor.

(3) Calculating the yield with the indicator diagram: this menu item function is used to calculate the oil well's yield and working status as well as self-correct the system.

(4) Data inquiry: this menu item function is used to inquire the oil well yield results, oil well data and oil extractor data.

(5) Data analysis: this menu item function is used to analyze the oil well yield, analyze the working status of the oil well and analyze system data, etc.

(6) Data alarm: this menu item function is used to analyze the oil extractor's status, trigger alarm against abnormal oil extractor status and stop the well from operating remotely. 


\section{SYSTEMATIC SUMMARY}

The use of the acceleration speed meter-type sensor to measure the polished rod's stroke and times of strokes and realize wireless measurement of displacement is this system's innovation. The technology could accurately measure the indicator diagram in a synchronized manner, offering true and reliable data resource for the application of the single-well metering software.

The system can automatically monitor the parameters of the indicator diagram as per specified collection period. Through the monitoring software and single-well metering software, the system could calculate and analyze the single-well output and diagnose the oil well's working status.

Through the double-volume, large-pot single variable comparison tests and debugging, the system could well collect data and conduct communication, offering accurate indicator diagram data, guaranteeing normal system operation and making sure every technical index achieves the requirement of design.

(1) According to on-site requirement, the collection time and interval of the ground indicator diagram data are defined. In data processing points, through the monitor and metering analysis software, parameter setting could be conducted directly.

(2) Realizing all-weather data collection

The load sensor would be installed on the wellhead of the oil extractor, and the angular position sensor would be installed on the walking beam of the oil extractor, respectively measuring the loading changes of the polished rod and the changes of the oil extractor's operating cycles. Upon signal transformation, the ground indicator diagram of the oil extractor can be detected.

(3) Realizing remote transmission of the collected data

The actually measured indicator diagram data of the oil well at each data collection point would be sent to the data processing points through data transmission radio station. The data processing points could directly observe the test indicator diagram and working status of each oil well. Upon abnormal occurrences management persons could be timely reminded to take corresponding measures. Meanwhile, the system would automatically store the collected data into the database.

(4) Realizing automatic metering of the liquid yield of the oil well

Through the indicator diagram oil well metering analysis software, the data collected by the ground indicator diagram could be analyzed to facilitate working status analysis and the liquid yield of the oil well.

(5) Realizing automatic oil well management

The system establishes the database of the oil well and of the indicator diagram and other relevant database and realizes the inquiry of the indicator diagram data, the liquid yield calculation of the oil well and the generation of the production statements. The oil well's liquid yield could be directly reported where internet access is available.

\section{REFERENCES}

[1] Gao Guohua, Peng You, Yu Guo'an: Qualitative Analysis Method of the Indicator Diagram of Sucker Rod Pumping Wells. [J] Journal of Petroleum, 1993

[2] Yan Changliang, Peng Yong: Technological Study on Pump Indicator Diagram Single Well's Automatic Oil Yield Measurement Journal of Xi'an Petroleum Institute, 2006

[3] Li Shen: Optimized Design of the Oil Extraction System of the Sucker Rod Pump. Dalian University of Technology, 2005 